\title{
Probabilistic Analysis of Pressurized Tunnels against Face Stability Using Collocation-Based Stochastic Response Surface Method
}

\author{
Guilhem Mollon ${ }^{1}$; Daniel Dias²; and Abdul-Hamid Soubra, M.ASCE ${ }^{3}$
}

\begin{abstract}
A probabilistic analysis of the face stability of a tunnel driven by a compressed-air pressurized shield is presented. The collocation-based stochastic response surface methodology (CSRSM) is used. The deterministic model employed in the probabilistic analysis is analytical. A translational multiblock collapse mechanism in the framework of the kinematic theorem of limit analysis forms the basis of the analysis. The soil friction angle and cohesion are considered as random variables. CSRSM was first validated by the comparison of the results obtained from the original analytical deterministic model. Then, the influence of the probabilistic characteristics of the uncertain variables was studied. Contrary to the correlation between $c$ and $\varphi$ and the coefficients of variation of these variables, which have a significant effect on the variability of the critical collapse pressure, the nonnormality of the distributions of the random variables was shown not to have a considerable effect on the distribution of the output. DOI: 10.1061/(ASCE)GT.1943-5606.0000443. (C) 2011 American Society of Civil Engineers.
\end{abstract}

CE Database subject headings: Tunnels; Tunneling; Probability; Reliability; Failures; Stochastic processes.

Author keywords: Tunnel; Face stability; Shield tunneling; Probabilistic methods; Reliability; Failure probability.

\section{Introduction}

The face stability analysis of a circular tunnel driven by a pressurized shield is of considerable interest. It requires the determination of a so-called "critical collapse pressure" of the tunnel face (denoted $\sigma_{c}$ ); that is, the smallest applied pressure necessary to prevent soil collapse. This issue has been extensively studied by several writers in the case of purely cohesive soils (Broms and Bennermark 1967; Davis et al. 1980; Ellstein 1986; Augarde et al. 2003; Klar et al. 2007; among others) and in the case of frictional soils with or without cohesion (Leca and Dormieux 1990; Chambon and Corté 1994; Eisenstein and Ezzeldine 1994; Anagnostou and Kovari 1996; Mollon et al. 2009a, b, 2010; among others). All studies cited except those by Mollon et al. (2009a, b) were deterministic. A probabilistic approach would be an improvement on the traditional deterministic methods because it would account for the inherent uncertainties of the input parameters in a rational way. In this paper, the uncertain parameters are modeled as random variables.

${ }^{1} \mathrm{Ph} . \mathrm{D}$. Student, INSA Lyon, Université de Lyon, LGCIE Domaine B, Bât. J.C.A. Coulomb, Domaine scientifique de la Doua, 69621 Villeurbanne cedex, France (corresponding author). E-mail: Guilhem .Mollon@insa-lyon.fr

${ }^{2}$ Associate Professor, INSA Lyon, Université de Lyon, LGCIE Domaine B, Bât. J.C.A. Coulomb, Domaine scientifique de la Doua, 69621 Villeurbanne cedex, France. E-mail: Daniel.Dias@insa-lyon.fr

${ }^{3}$ Professor, Univ. of Nantes, Institut de Recherche en Génie Civil et Mécanique, UMR CNRS 6183, Bd. de l'université, BP 152, 44603 Saint-Nazaire cedex, France. E-mail: Abed.Soubra@univ-nantes.fr

Note. This manuscript was submitted on January 8, 2010; approved on August 27, 2010; published online on September 18, 2010. Discussion period open until September 1, 2011; separate discussions must be submitted for individual papers. This paper is part of the Journal of Geotechnical and Geoenvironmental Engineering, Vol. 137, No. 4, April 1, 2011. CASCE, ISSN 1090-0241/2011/4-385-397/\$25.00.
The most robust probabilistic approach is the Monte Carlo(MC-) simulation method. This method requires a large number of calls of the deterministic model, especially for small values of the failure probability (e.g., approximately 1,000,000 samples for a failure probability of $10^{-5}$ ). Although deterministic numerical methods such as those derived from the FEM or the finitedifference method (FDM) are appealing for determining the critical collapse pressure because they do not require an assumption about the shape of the collapse mechanism, these methods are difficult to use in a probabilistic framework, particularly when using the MC-simulation method. They require a long time to compute. This issue is of great concern when dealing with three-dimensional (3D) simulations. To overcome this shortcoming, the collocation-based stochastic response surface method (CSRSM) may be used. CSRSM replaces the complex numerical model with a metamodel - an analytical function of the input parameters - and performs the Monte Carlo probabilistic analysis on this metamodel. This analytical function is called polynomial chaos expansion (PCE). For this paper, a deterministic, not time-consuming, analytical model presented extensively in Mollon et al. (2009a) was used to validate CSRSM. This was done (1) by comparing the Monte Carlo probabilistic results obtained by using the original analytical model with the ones obtained by using the metamodel; and (2) by comparing the response surfaces given by the original analytical model and the metamodel. This validation allowed the use of CSRSM with confidence in complex finite-element or finite-difference models for which the model analytical equation was not available. After this validation, a global sensitivity analysis was performed to determine the variables that had a significant effect on the variability of the system response. Also, a parametric study was performed to evaluate the effect of the statistical parameters of the uncertain variables [i.e., coefficient of variation (COV), correlation, and type of probability distribution] on both (1) the distribution of the critical collapse pressure; and (2) the failure probabilities corresponding to different values of the tunnel applied pressure. 


\section{CSRSM}

CSRSM is a general and powerful method (Isukapalli et al. 1998; Isukapalli 1999; Berveiller et al. 2006; Sudret et al. 2006; Sudret 2008; Phoon and Huang 2007; Huang et al. 2009) that determines a single value for the failure probability of a system for a given value of the applied load, as is the case in classical response surface methodology (Mollon et al. 2009a, b; Youssef Abdel Massih and Soubra 2008; Youssef Abdel Massih et al. 2008). It also determines the full probability distribution function (PDF) of the system response (ultimate load) by accounting for the uncertainties of the input of uncertain parameters by their probability distributions. The PDF provides information on the mean, variance, and other statistical moments (e.g., skewness and kurtosis) of the system response. It also computes the failure probabilities for different values of the applied load (i.e., at the distribution tail).

CSRSM determines the propagation of uncertainties from the input data to the system output (i.e., the system response) for a given mechanical model. In this method, the deterministic model is considered a black box. It is not necessary to modify the existing deterministic model, which may be derived from analytical equations or numerical simulations by using, for example, FEM or FDM. Instead, determine the response of the mechanical model at a given number of collocation points (i.e., for different values of the input parameters). The primary assumption in CSRSM is that the output is approximated in the standard space of random variables by a PCE, whose unknown coefficients are determined either by a regression approach or by a projection method. In this paper, a regression approach was used. The determination of the probability distribution of the system response may be summarized by the following:

- Choose the order of the polynomial chaos and determine the pattern (i.e., number and positions) of the collocation points. The available number of collocation points and their positions in the standard space of random variables depend on the chosen order of the chaos and the number of the random variables. The choice of the optimal chaos order is discussed subsequently in this paper;

- Transform the collocation points from the standard to the physical space of random variables;

- Compute the output (i.e., system response) corresponding to each collocation point in the physical space by using the deterministic mechanical model;

- Determine the values of the unknown coefficients of the PCE by using a linear system of equations; and

- Compute the PDF of the approximated system response by performing the Monte Carlo-simulation method on the PCE determined previously, and deduce the statistical moments of this distribution.

Although CSRSM is primarily devoted to the assessment of the probability distribution function of the system response, the determination of PCE in CSRSM has another great practical advantage: it determines the failure probabilities at the distribution tail by using the obtained PCE.

\section{Probabilistic Analysis of Tunnel Face Stability}

A Monte Carlo probabilistic analysis of the face stability of a circular tunnel driven by a compressed-air pressurized shield is discussed in this section for the purposes of determining (1) the probability distribution of the tunnel face collapse pressure $\sigma_{c}$ (i.e., system response); and (2) the failure probabilities for the practical values of the applied pressure (i.e., at the distribution tail). CSRSM is used for performing the Monte Carlo probabilistic analysis. A quite simple analytical model derived from the kinematical approach of limit analysis is used to determine the values of the tunnel face collapse pressure at different collocation points. In this paper, only two input variables were considered as random: the soil friction angle $\varphi$ and the soil cohesion $c$. These two variables were characterized by their respective probability distribution function: a normal or beta distribution was assumed for $\varphi$ and a normal or lognormal distribution was assumed for $c$. Their respective means and standard deviations values are denoted $\mu_{\varphi}, \mu_{c}$ and $\sigma_{\varphi}, \sigma_{c}$, respectively. In the event of correlated random variables, their correlation coefficient is denoted $\rho_{\varphi c}$. In subsequent sections, the deterministic model is first presented. It is followed by a brief description of CSRSM as applied to the present problem of tunnel face collapse pressure involving two random variables.

\section{Deterministic Model}

The problem of a computation of the tunnel face collapse pressure $\sigma_{c}$ can be idealized, as shown in Fig. 1, by considering a circular rigid tunnel of diameter $D$ driven under a depth of cover $C$. The active collapse of the tunnel face is triggered by the application of surcharge $\sigma_{s}$ and soil unit weight $\gamma$, with the tunnel face pressure $\sigma_{c}$ providing resistance against failure. The critical collapse pressure is a function of the strength parameters of the soil, namely the cohesion $c$ and the friction angle $\varphi$. The issue of the choice of the correct values of the shear strength parameters for a soil is rather complex. It has been stated (Chen 1975; Chen and Liu 1990) that the failures involving large zones of a soil mass may not occur suddenly, but that an assumption of a progressive failure is probably closer to reality. This is because of the possibility of the elastic deformation of the soil before global failure, which can lead some zones of the soil to fail locally while the strain level of other zones is not high enough for the soil to fail. This assumption implies that the strain level is not constant over the discontinuity surfaces and it should vary a lot on these surfaces depending on how the entire failure develops. For materials that exhibit a postpeak strain softening behavior (e.g., dense sands and overconsolidated clays), the perfectly plastic representation of its ultimate behavior is only acceptable in an average sense. For this reason, the method for idealizing the plastic behavior of such a material by using the peak characteristic is incorrect and could lead to unconservative designs. The average mobilized stress level over the discontinuity surfaces at the instant of collapse therefore, should be somewhere among the

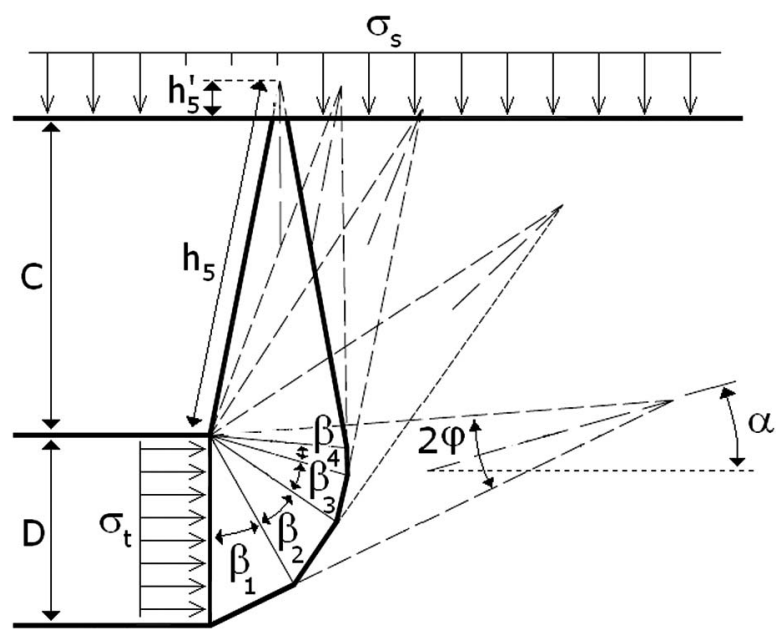

Fig. 1. Cross-section of the proposed multiblock failure mechanism for the face stability analysis 
peak stress located on areas that fail the last, triggering the global failure, and the residual stress located at the zones that failed earlier, having a large strain level at the moment of global failure. The choice of the correct values of $\varphi$ and $c$ in any large-scale stability analysis is quite complex and left to the judgment of expert geotechnical engineers.

Several theoretical models have been presented in literature for the computation of the tunnel face collapse pressure. Mollon et al. (2009a) have considered a three-dimensional multiblock failure mechanism in the framework of the kinematical approach in limit analysis. Their mechanism constitutes an improvement of the twoblock mechanism by Leca and Dormieux (1990) because it allows the three-dimensional slip surface to develop more freely because of its great degree of freedom. This mechanism will be used in this paper for the probabilistic analysis. A brief description of this failure mechanism is given subsequently. For more details, refer to Mollon et al. (2009a).

The multiblock failure mechanism is composed of several truncated rigid cones with circular cross sections and with opening angles equal to $2 \varphi$. A mechanism with only five blocks is considered in this paper, as shown in Fig. 1, because the increase in the number of blocks does not significantly improve the accuracy of the solution. The upper rigid cone will or will not intersect the ground surface depending on $\varphi$ and $C / D$ values. For simplicity, only the case of a nonoutcropping mechanism is considered in this paper. This corresponds to $C / D>2$ and $\varphi>10^{\circ}$. The usual values of $\varphi$ and $C / D$ belong to this category of nonoutcropping mechanisms. The multiblock mechanism is a translational kinematically admissible failure mechanism. The different truncated conical blocks move as rigid bodies. These truncated rigid cones translate with velocities of different directions, which are collinear with the cones axes and make an angle $\varphi$ with the conical discontinuity surfaces to respect the normality condition required by the limit analysis theory. This mechanism is completely defined by five angular parameters: $\alpha, \beta_{1}, \beta_{2}, \beta_{3}$, and $\beta_{4}$. By equating the total rate of energy dissipation to the total rate of external work, it is possible to compute the collapse pressure $\sigma_{c}$. Because the face pressure is resisting collapse, it should be considered a negative load. The optimal solution that the mechanism can provide in the framework of the kinematical approach is obtained by the minimization of $\left(-\sigma_{c}\right)$ to the five angular parameters of the failure mechanism. This is equivalent to a maximization of $\sigma_{c}$.

A comparison of the deterministic results provided by the proposed mechanism and the numerical model used in Mollon et al. (2009b) was proposed in Mollon et al. (2009a) and showed a satisfying agreement in the trends. More precisely, the present mechanism underestimates the critical collapse pressure by $10-20 \%$ with respect to the numerical model. The proposed mechanism is not the one leading to the most critical collapse pressure in the framework of the kinematical approach, as was demonstrated in Mollon et al. (2010). Nevertheless, it was chosen for this study because its small computational cost makes possible the validation of CSRSM. This is done by comparison of a great number of Monte Carlo-simulations with the original analytical model. CSRSM is not expected to be used in the future with this model but with much more complex and time-consuming numerical models. The question of whether this model is very accurate or not for the determination of the collapse pressure is of little interest in this paper.

\section{PCE and Collocation Points}

The two random variables adopted in this analysis (i.e., $\varphi$ and $c$ ) have to be represented in the PCE by two standard variables: $\xi_{1}$ and $\xi_{2}$, respectively. For a prescribed PCE order, the available collocation points can be easily determined in the standard space of random variables. Each standard variable ( $\xi_{1}$ or $\xi_{2}$, in this case) can take the values of the roots of the univariate Hermite polynomial of order $n+1$ where $n$ is the order of the polynomial chaos. Thus, the available collocation points are those that result from all possible combinations of the roots for each standard random variable. The univariate Hermite polynomials are given in Appendix I. Table 1 provides the roots, the general shape of the PCE that will approximate the response of the model (i.e., $\sigma_{c}$ in this paper), the number $p$ of unknown coefficients, and the number $M$ of available collocation points, for the case of two random variables and for different values of the order $n$ of the polynomial chaos $(n=2,3,4,5)$. For a PCE of order $n$, and for $n_{v}$ random variables, the number $p$ of unknown PCE coefficients and the number $M$ of available collocation points are given by (Isukapalli 1999)

$$
p=\frac{\left(n+n_{v}\right) !}{n ! n_{v} !}
$$

$$
M=(n+1)^{n_{v}}+ \begin{cases}0 & \text { if one of the roots of the }(n+1) \text { th univariate Hermite polynomial is } 0 \\ 1 & \text { otherwise }\end{cases}
$$

As shown in Eq. (2), Isukapalli (1999) proposed to increase the number of collocation points by one to include the origin of the standard space in the event that the univariate Hermite polynomial of order $n+1$ does not include a root equal to zero. This contingency is necessary because the point is located in the region of maximum probability (Phoon and Huang 2007). The $\Gamma$ terms in Table 1 are multidimensional Hermite polynomials. Their expressions are given in Appendix I. In Table 1, the number of available collocation points is greater than the number of unknown coefficients for all the orders. It is desirable, if possible, to compute the value of the system response at a number of collocation points equal to approximately twice the number of unknown coefficients (Isukapalli 1999). A better approximation of the response is obtained when using a regression approach. The choice of the different collocation points among those available should respect symmetry with respect to the origin of the standard space, and the chosen collocation points should be located as close as possible to the origin of the standard space (Isukapalli 1999). In this paper, in cases for which only two random variables were used, the total number $M$ of the available collocation points was considered in the analysis because $2 p>M$ whatever is the value of the chaos order.

\section{Transformation of Collocation Points from Standard to Physical Space}

For each chosen point (i.e., $\left.\xi_{1, m}, \xi_{2, m}\right)$ for which $m=1, \ldots, 2 p$, determine the corresponding point (i.e., $\varphi_{m}, c_{m}$ ) to be introduced 
Table 1. Two-Variable PCEs Used for Different Values of the PCE Order

\begin{tabular}{|c|c|c|c|c|}
\hline$n$ & $\begin{array}{l}\text { Roots of univariate Hermite } \\
\text { polynomials of order } n+1\end{array}$ & Expression of PCEs for different orders & $p$ & $M$ \\
\hline 2 & $\{0 ; \pm \sqrt{3}\}$ & $\begin{aligned} U_{2}= & a_{0,0} \cdot \Gamma_{0,0}+a_{1,0} \cdot \Gamma_{1,0}\left(\xi_{1}\right)+a_{0,1} \cdot \Gamma_{0,1}\left(\xi_{2}\right)+a_{2,0} \cdot \Gamma_{2,0}\left(\xi_{1}\right) \\
& +a_{1,1} \cdot \Gamma_{1,1}\left(\xi_{1}, \xi_{2}\right)+a_{0,2} \cdot \Gamma_{0,2}\left(\xi_{2}\right)\end{aligned}$ & 6 & 9 \\
\hline 3 & $\{ \pm \sqrt{3 \pm \sqrt{6}}\}$ & $\begin{aligned} U_{3}= & a_{0,0} \cdot \Gamma_{0,0}+a_{1,0} \cdot \Gamma_{1,0}\left(\xi_{1}\right)+a_{0,1} \cdot \Gamma_{0,1}\left(\xi_{2}\right)+a_{2,0} \cdot \Gamma_{2,0}\left(\xi_{1}\right) \\
& +a_{1,1} \cdot \Gamma_{1,1}\left(\xi_{1}, \xi_{2}\right)+a_{0,2} \cdot \Gamma_{0,2}\left(\xi_{2}\right)+a_{3,0} \cdot \Gamma_{3,0}\left(\xi_{1}\right) \\
& +a_{2,1} \cdot \Gamma_{2,1}\left(\xi_{1}, \xi_{2}\right)+a_{1,2} \cdot \Gamma_{1,2}\left(\xi_{1}, \xi_{2}\right)+a_{0,3} \cdot \Gamma_{0,3}\left(\xi_{2}\right)\end{aligned}$ & 10 & $16+1$ \\
\hline 4 & $\{0 ; \pm \sqrt{5 \pm \sqrt{10}}\}$ & $\begin{aligned} U_{4}= & a_{0,0} \cdot \Gamma_{0,0}+a_{1,0} \cdot \Gamma_{1,0}\left(\xi_{1}\right)+a_{0,1} \cdot \Gamma_{0,1}\left(\xi_{2}\right)+a_{2,0} \cdot \Gamma_{2,0}\left(\xi_{1}\right) \\
& +a_{1,1} \cdot \Gamma_{1,1}\left(\xi_{1}, \xi_{2}\right)+a_{0,2} \cdot \Gamma_{0,2}\left(\xi_{2}\right)+a_{3,0} \cdot \Gamma_{3,0}\left(\xi_{1}\right) \\
& +a_{2,1} \cdot \Gamma_{2,1}\left(\xi_{1}, \xi_{2}\right)+a_{1,2} \cdot \Gamma_{1,2}\left(\xi_{1}, \xi_{2}\right)+a_{0,3} \cdot \Gamma_{0,3}\left(\xi_{2}\right) \\
& +a_{4,0} \cdot \Gamma_{4,0}\left(\xi_{1}\right)+a_{3,1} \cdot \Gamma_{3,1}\left(\xi_{1}, \xi_{2}\right)+a_{2,2} \cdot \Gamma_{2,2}\left(\xi_{1}, \xi_{2}\right) \\
& +a_{1,3} \cdot \Gamma_{1,3}\left(\xi_{1}, \xi_{2}\right)+a_{0,4} \cdot \Gamma_{0,4}\left(\xi_{2}\right)\end{aligned}$ & 15 & 25 \\
\hline 5 & $\{ \pm 3.324257 ; \pm 1.889176 ; \pm 0.616707\}$ & $\begin{aligned} U_{5}= & a_{0,0} \cdot \Gamma_{0,0}+a_{1,0} \cdot \Gamma_{1,0}\left(\xi_{1}\right)+a_{0,1} \cdot \Gamma_{0,1}\left(\xi_{2}\right)+a_{2,0} \cdot \Gamma_{2,0}\left(\xi_{1}\right) \\
& +a_{1,1} \cdot \Gamma_{1,1}\left(\xi_{1}, \xi_{2}\right)+a_{0,2} \cdot \Gamma_{0,2}\left(\xi_{2}\right)+a_{3,0} \cdot \Gamma_{3,0}\left(\xi_{1}\right) \\
& +a_{2,1} \cdot \Gamma_{2,1}\left(\xi_{1}, \xi_{2}\right)+a_{1,2} \cdot \Gamma_{1,2}\left(\xi_{1}, \xi_{2}\right)+a_{0,3} \cdot \Gamma_{0,3}\left(\xi_{2}\right) \\
& +a_{4,0} \cdot \Gamma_{4,0}\left(\xi_{1}\right)+a_{3,1} \cdot \Gamma_{3,1}\left(\xi_{1}, \xi_{2}\right)+a_{2,2} \cdot \Gamma_{2,2}\left(\xi_{1}, \xi_{2}\right) \\
& +a_{1,3} \cdot \Gamma_{1,3}\left(\xi_{1}, \xi_{2}\right)+a_{0,4} \cdot \Gamma_{0,4}\left(\xi_{2}\right)+a_{5,0} \cdot \Gamma_{5,0}\left(\xi_{1}\right) \\
& +a_{4,1} \cdot \Gamma_{4,1}\left(\xi_{1}, \xi_{2}\right)+a_{3,2} \cdot \Gamma_{3,2}\left(\xi_{1}, \xi_{2}\right)+a_{2,3} \cdot \Gamma_{2,3}\left(\xi_{1}, \xi_{2}\right) \\
& +a_{1,4} \cdot \Gamma_{1,4}\left(\xi_{1}, \xi_{2}\right)+a_{0,5} \cdot \Gamma_{0,5}\left(\xi_{2}\right)\end{aligned}$ & 21 & $36+1$ \\
\hline
\end{tabular}

Note: $n=$ order of the PCE, $p=$ number of unknown PCE coefficients, and $M=$ number of available collocation points.

in the deterministic model. If a correlation exists between the random variables $c_{m}$ and $\varphi_{m}$, correlate the standard variables by multiplying the vector of standard uncorrelated variables by matrix $\mathrm{H}$ which is the Cholesky transform of the correlation matrix $\Sigma$. In the case of two random variables, we have

$$
\left[\begin{array}{l}
\xi_{1 C, m} \\
\xi_{2 C, m}
\end{array}\right]=\mathrm{H} \cdot\left[\begin{array}{l}
\xi_{1, m} \\
\xi_{2, m}
\end{array}\right]
$$

where $\xi_{1 C, m}, \xi_{2 C, m}=$ standard correlated collocation point corresponding to $\xi_{1, m}, \xi_{2, m}$. The standard correlated variables must be transformed into the physical correlated variables in conformity with the marginal distribution of each variable by using the equation $\mathrm{X}=F^{-1}[\Phi(\xi)]$ where $\mathrm{X}=$ physical random variable; $\xi=$ standard normal variable; and $F(\cdot), \Phi(\cdot)=$ corresponding CDFs. In this case

$$
\begin{cases}\varphi_{m}=F_{\varphi}^{-1} & {\left[\Phi\left(\xi_{1 C, m}\right)\right]} \\ c_{m}=F_{c}^{-1} & {\left[\Phi\left(\xi_{2 C, m}\right)\right]}\end{cases}
$$

To conclude, for the chosen collocation points in the standard space, it is possible to find the corresponding points in the physical space and to call the deterministic model for each of these points to determine the corresponding system responses. Fig. 2 shows the effect of correlation or nonnormality of random variables on the position of the available collocation points in the physical space. For the reference case of normal uncorrelated variables, the nonnormality cancels the symmetry with respect to the mean value, and the correlation creates a distortion of the entire pattern of the collocation points.In the standard space, the position of the collocations points is unique regardless of the assumptions about correlation or nonnormality.

\section{Computation of PCE Coefficients}

Because the multidimensional Hermite polynomials $\Gamma$ form an orthogonal basis, the $p$ unknown coefficients of the polynomial chaos could be determined by using only $p$ calls of the deterministic model and by solving a simple linear system of dimension $p$. In practice, this method quite unstable, especially when using a high order PCE (Isukapalli 1999). As previously mentioned, a number $m$ of simulation results or collocation points that is not too far from $2 p$, (i.e., $m \cong 2 p$ ) was suggested by Isukapalli (1999). Write the matrix $\mathrm{N}$ of dimensions $(m \times p)$ in which each column is relative to a given coefficient and each line is relative to a call of the deterministic model. As an illustration, the $\mathrm{N}$ matrix for a second order PCE and two random variables is as follows (the matrix corresponding to other PCE orders is straightforward):

$$
\mathbf{N}=\left[\begin{array}{cccccc}
\Gamma_{0,0} & \Gamma_{1,0}\left(\xi_{1,1}\right) & \Gamma_{0,1}\left(\xi_{2,1}\right) & \Gamma_{2,0}\left(\xi_{1,1}\right) & \Gamma_{1,1}\left(\xi_{1,1}, \xi_{2,1}\right) & \Gamma_{0,2}\left(\xi_{2,1}\right) \\
\Gamma_{0,0} & \Gamma_{1,0}\left(\xi_{1,2}\right) & \Gamma_{0,1}\left(\xi_{2,2}\right) & \Gamma_{2,0}\left(\xi_{1,2}\right) & \Gamma_{1,1}\left(\xi_{1,2}, \xi_{2,2}\right) & \Gamma_{0,2}\left(\xi_{2,2}\right) \\
\cdot & \cdot & \cdot & \cdot & \cdot & \cdot \\
\cdot & \cdot & \cdot & \cdot & \cdot & \cdot \\
\cdot & \cdot & \cdot & \cdot & \cdot & \cdot \\
\Gamma_{0,0} & \Gamma_{1,0}\left(\xi_{1, m}\right) & \Gamma_{0,1}\left(\xi_{2, m}\right) & \Gamma_{2,0}\left(\xi_{1, m}\right) & \Gamma_{1,1}\left(\xi_{1, m}, \xi_{2, m}\right) & \Gamma_{0,2}\left(\xi_{2, m}\right)
\end{array}\right]
$$

If $\underline{a}$ is the column vector of the coefficients $a_{i}$ ( $p$ terms), and $f$ is the column vector of the model responses (i.e., $m$ values of the critical collapse pressure), the coefficients of the PCE can be obtained by solving the following linear system:

$$
\underline{N^{t}} \cdot \underline{N} \cdot \underline{a}=\underline{N}^{t} \cdot \underline{f}
$$

For a simpler use of some mathematical formulas presented subsequently in this paper, the multivariate Hermite polynomials $\Gamma_{i, j}$ are often called $\psi_{k}$ where $k$ is a single index corresponding 


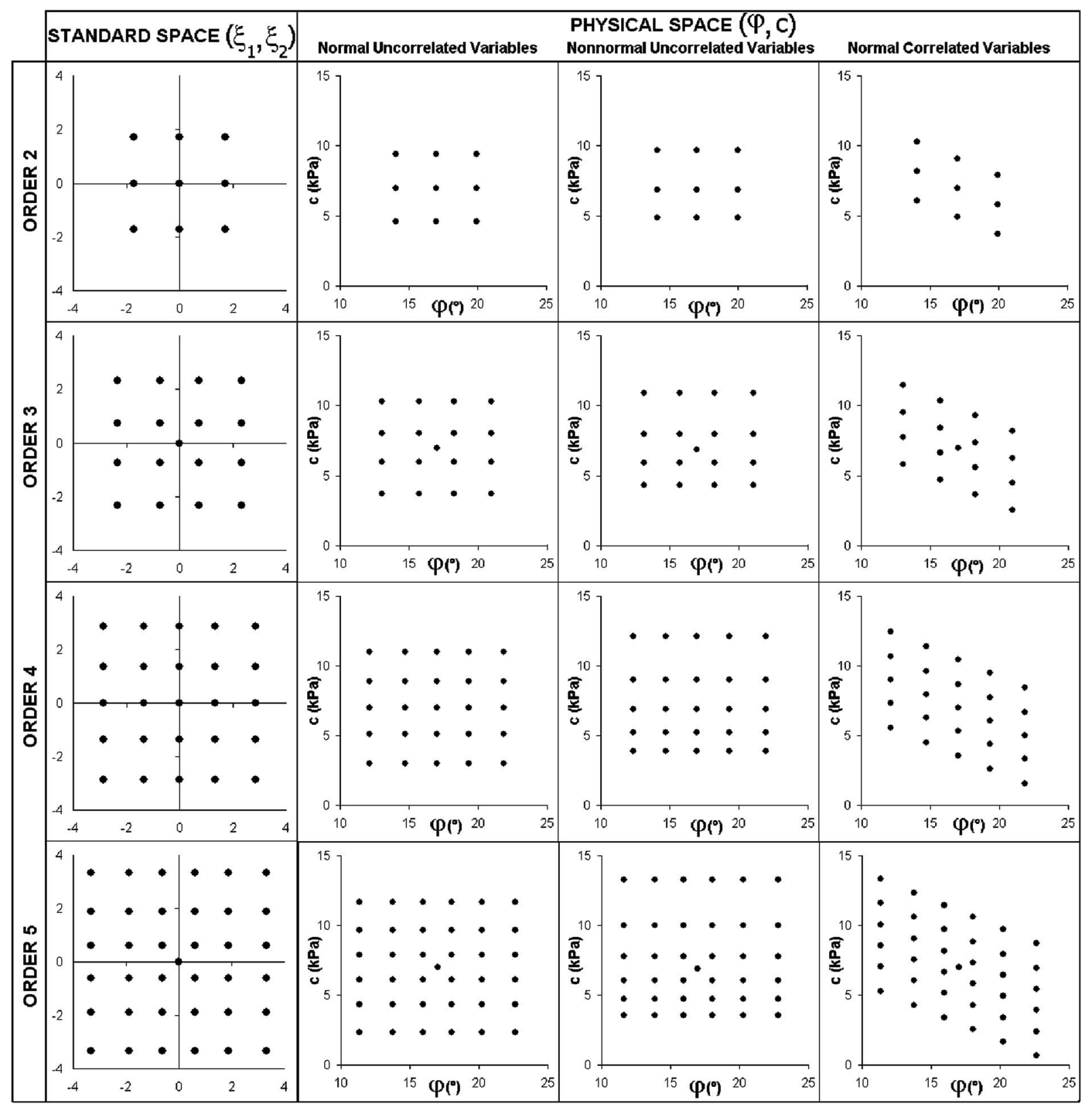

Fig. 2. Position of the collocation points in the standard space and their corresponding positions in the physical space depending on normality and correlation of the variables for several PCE orders

to one vector in the PCE basis (see Appendix I for more details).

\section{Computation of Output Characteristics}

Once the PCE coefficients have been determined, the Monte Carlosimulation method can be used to plot the histogram of the system response and the corresponding PDF. Either generate a large number [e.g., $\left.10^{6}\right)$ of samples $\left.\left(\xi_{1}, \xi_{2}\right)\right]$ for the histogram to be regular and directly transformed into a PDF, or use a reduced number (e.g., 100,000) of samples and the kernel smoothing technique to directly plot the PDF. The kernel smoothing technique was used for this paper. It states that an estimator of the PDF at a point $x$ is given by

$$
f(x, h)=\frac{1}{\mathrm{~N}_{\text {sample }} \cdot h} \sum_{i=1}^{\mathrm{N}_{\text {sample }}} \mathrm{K}\left(\frac{x-\mathrm{X}_{i}}{h}\right)
$$

where $\mathrm{X}_{i}=$ system response for sample $i$ (i.e., the approximated response given by the PCE for the $i$ th sample); $h=$ bandwidth; and $\mathrm{K}=$ function called kernel. In this study, a Gaussian kernel function was used. The Monte Carlo-simulation method employed in this paper is not a highly time-consuming method because it does not make use of the real deterministic model. Only its approximation by its PCE is used. From the Monte Carlo-simulation technique, it is also possible to obtain estimators of the first four statistical moments of the response (i.e., mean $\bar{\mu}$, variance $\bar{\sigma}^{2}$, skewness $\bar{\delta}_{s}$, and kurtosis $\bar{\kappa}_{s}$ ) by using Matlab or any other commercial software application. The resulting statistical moments are only estimators, and it would be better to compute them analytically. This is possible when using CSRSM because formulas exist that provide the statistical moments analytically from the PCE coefficients as follows (Berveiller et al. 2006):

$$
\mu=a_{0}
$$




$$
\begin{gathered}
\sigma^{2}=\sum_{i=1}^{p-1} \mathrm{E}\left(\psi_{i}^{2}\right) \cdot a_{i}^{2} \\
\delta_{s}=\frac{1}{\sigma_{s}^{3}} \sum_{i=1}^{p-1} \sum_{j=1}^{p-1} \sum_{k=1}^{p-1} \mathrm{E}\left(\psi_{i} \cdot \psi_{j} \cdot \psi_{k}\right) \cdot a_{i} \cdot a_{j} \cdot a_{k} \\
\kappa_{s}=\frac{1}{\sigma_{s}^{4}} \sum_{i=1}^{p-1} \sum_{j=1}^{p-1} \sum_{k=1}^{p-1} \sum_{l=1}^{p-1} \mathrm{E}\left(\psi_{i} \cdot \psi_{j} \cdot \psi_{k} \cdot \psi_{l}\right) \cdot a_{i} \cdot a_{j} \cdot a_{k} \cdot a_{l}
\end{gathered}
$$

In these equations, the method of computation of $\mathrm{E}\left(\psi_{i}^{2}\right)$, $\mathrm{E}\left(\psi_{i} \cdot \psi_{j} \cdot \psi_{k}\right)$, and $\mathrm{E}\left(\psi_{i} \cdot \psi_{j} \cdot \psi_{k} \cdot \psi_{l}\right)$ is given in Appendix II, Eqs. (8)-(11) provide very fast results for the moments if the number $p$ of the unknown coefficients remains small (i.e., for a small PCE order and for a small number of variables). On the contrary, the analytical computation of the skewness and of the kurtosis [Eqs. (10) and (11)] can become very time-consuming if the number $p$ of coefficients is important because of the number of terms involved in the summations [e.g., 160,000 terms, i.e., $(p-1)^{4}=$ $(21-1)^{4}$ for the computation of $\kappa_{s}$ in the case of two random variables and a polynomial chaos of the fifth order]. In such cases, it is more efficient to perform a Monte Carlo-sampling with a sufficiently high number of samples for the estimators to be accurate (e.g., 1,000,000 samples). In this paper, the statistical moments were calculated analytically.

Finally, a global sensitivity analysis of the system response can be performed analytically from PCE coefficients by computing Sobol indexes of each random variable and group of random variables (Sudret 2008). The Sobol index of a variable or a group of variables provides the contribution of the uncertainty for this variable or group of variables to the total variance of the response. Subsequently, the sum of all the Sobol indexes of a model is equal to 1. For the computation of the Sobol indexes, reorder the terms of the PCE, and gather them into categories involving a unique random variable such as $\xi_{1}$ or $\xi_{2}$ or a group of random variables such as $\xi_{1}, \xi_{2}$, and so on. For example, for the fourth order PCE with two random variables (cf. Table 1), by reordering the terms following the three categories $\left(\xi_{1}, \xi_{2}\right.$, and $\left.\xi_{1}, \xi_{2}\right)$, it is possible to obtain

$$
\begin{aligned}
U_{4}= & a_{0,0} \cdot \Gamma_{0,0}+\left[a_{1,0} \cdot \Gamma_{1,0}\left(\xi_{1}\right)+a_{2,0} \cdot \Gamma_{2,0}\left(\xi_{1}\right)\right. \\
& \left.+a_{3,0} \cdot \Gamma_{3,0}\left(\xi_{1}\right)+a_{4,0} \cdot \Gamma_{4,0}\left(\xi_{1}\right)\right]+\left[a_{0,1} \cdot \Gamma_{0,1}\left(\xi_{2}\right)\right. \\
& \left.+a_{0,2} \cdot \Gamma_{0,2}\left(\xi_{2}\right)+a_{0,3} \cdot \Gamma_{0,3}\left(\xi_{2}\right)+a_{0,4} \cdot \Gamma_{0,4}\left(\xi_{2}\right)\right] \\
& +\left[a_{1,1} \cdot \Gamma_{1,1}\left(\xi_{1}, \xi_{2}\right)+a_{1,2} \cdot \Gamma_{1,2}\left(\xi_{1}, \xi_{2}\right)\right. \\
& +a_{2,1} \cdot \Gamma_{2,1}\left(\xi_{1}, \xi_{2}\right)+a_{3,1} \cdot \Gamma_{3,1}\left(\xi_{1}, \xi_{2}\right) \\
& \left.+a_{1,3} \cdot \Gamma_{1,3}\left(\xi_{1}, \xi_{2}\right)+a_{2,2} \cdot \Gamma_{2,2}\left(\xi_{1}, \xi_{2}\right)\right]
\end{aligned}
$$

The Sobol indexes can then be computed for each variable or group of variables as follows:

$$
\mathrm{SU}_{\alpha}=\frac{\sum a_{\alpha}^{2} \cdot \mathrm{E}\left(\psi_{\alpha}^{2}\right)}{\sigma^{2}}
$$

where

$$
\sigma^{2}=\sum a^{2} \cdot \mathrm{E}\left(\psi^{2}\right)
$$

In Eqs. (13) and (14), the index $\alpha$ indicates that the sum is made only for the terms including either $\left(\xi_{1}\right),\left(\xi_{2}\right)$, or $\left(\xi_{1}, \xi_{2}\right)$; that is, for one of the three brackets that appear in Eq. (12). The computation of $\mathrm{E}\left(\psi^{2}\right)$ is detailed in Appendix II. It can be proved easily that the sum of the Sobol indexes of all the variables and groups of variables is equal to 1 . Methods for computing Sobol indexes from a Monte Carlo-sampling exist (Sudret 2008), but the proposed analytical method provides exact values for an almost negligible computation time, whatever the PCE order and the number of variables are. This method is used in this paper for the study of the effect of the uncertainties of the different random variables.

\section{Validation of CSRSM}

This section aims at validating CSRSM by comparing the Monte Carlo probabilistic results provided by PCE with those obtained with the original analytical model. The results given by the analytical model will be called analytical results because they make use of the analytical equations of the mechanism and consider the exact analytical limit state surfaces. Normal uncorrelated variables are considered in this section. The illustrative values of the statistical moments of the random variables considered in this section will be referred to in this paper as the reference values. They are given as follows: $\mu_{\varphi}=17^{\circ}$ and $\operatorname{COV}(\varphi)=10 \% ; \mu_{c}=7 \mathrm{kPa}$ and $\operatorname{COV}(c)=20 \%$. The deterministic parameters used are $\gamma=$ $18 \mathrm{kN} / \mathrm{m}^{3}, D=10 \mathrm{~m}$ and $C / D>2$. All these values are typical of a stiff clay and a nonoutcropping mechanism.

The results of the Monte Carlo-simulations for both approaches (i.e., the metamodel and the original analytical model) were obtained by using 1,000,000 samples, although a significantly smaller number of samples could be suitable to plot the PDF because of the use of the kernel smoothing technique. These results are believed to be more accurate than those that would be obtained by the firstorder reliability method (FORM) because the curved and not the linearized limit state surfaces are used. In the following section, the optimal PCE order is determined first. It is followed by a comparison of the metamodel and the original analytical model for both failure probability and response surface. CSRSM validation is made in the following subsections for the reference case of a stiff clay corresponding to $\mu_{\varphi}=17^{\circ}$ and $\mu_{c} /(\gamma . D)=0.039$ and the critical collapse pressure is presented in the form of a dimensionless parameter $\sigma_{c} /(\gamma \cdot D)$.

\section{Optimal PCE Order in CSRSM}

The PCEs used in this section are successively of orders 2, 3, 4, and 5. The obtained PCE coefficients are given in Table 2. The convergence property of the PCE order is satisfied because the value of a PCE coefficient corresponding to a given $\psi_{i}$ tends to a constant value when the PCE order increases (see for example the lines corresponding to $\psi_{0}, \ldots, \psi_{5}$ in Table 2 . Each new order $n+1$ brings new terms in the PCE with respect to the previous order $n$, and the coefficients corresponding to the new terms decrease when $n$ increases. For example, from Table 2, some of the coefficients of the new terms brought by order 4 are much larger in absolute value than the ones brought by order 5 , which all are smaller than $10^{-3}$ in absolute value. Therefore, when the PCE order increases from 4 to 5 , the coefficients of the common terms remain stable because of the convergence property, and the coefficients of the new terms are very small. This indicates that the benefit of order 5 with respect to order 4 is small, although the number of collocation points and the computation time is $50 \%$ larger for order 5 than that of order 4 .

Table 3 presents the first four statistical moments of the critical collapse pressure computed analytically for different PCE orders. Almost no differences exist between the PCEs of orders 4 and 5 for the four moments, indicating that order 4 is sufficient to accurately compute them. Concerning the Sobol indexes, it appears that their 
Table 2. PCE Coefficients for Several Orders

\begin{tabular}{lccccc}
\hline & & \multicolumn{4}{c}{ PCE coefficients } \\
\cline { 2 - 6 } Term of the PCE & Order 2 & Order 3 & Order 4 & Order 5 \\
\hline$\Gamma_{0,0}$ & $\psi_{0}$ & 28,9212 & 28,9260 & 28,9220 & 28,9220 \\
$\Gamma_{1,0}\left(\xi_{1}\right)$ & $\psi_{1}$ & $-5,0035$ & $-4,8585$ & $-4,8496$ & $-4,8496$ \\
$\Gamma_{0,1}\left(\xi_{2}\right)$ & $\psi_{2}$ & $-4,6766$ & $-4,6277$ & $-4,6253$ & $-4,6266$ \\
$\Gamma_{2,0}\left(\xi_{1}\right)$ & $\psi_{3}$ & 0,6080 & 0,6335 & 0,6350 & 0,6353 \\
$\Gamma_{1,1}\left(\xi_{1}, \xi_{2}\right)$ & $\psi_{4}$ & 0,5005 & 0,5111 & 0,4901 & 0,4896 \\
$\Gamma_{0,2}\left(\xi_{2}\right)$ & $\psi_{5}$ & 0,0002 & $-0,0006$ & 0,0000 & 0,0000 \\
$\Gamma_{3,0}\left(\xi_{1}\right)$ & $\psi_{6}$ & & $-0,0754$ & $-0,0792$ & $-0,0796$ \\
$\Gamma_{2,1}\left(\xi_{1}, \xi_{2}\right)$ & $\psi_{7}$ & & $-0,0502$ & $-0,0518$ & $-0,0502$ \\
$\Gamma_{1,2}\left(\xi_{1}, \xi_{2}\right)$ & $\psi_{8}$ & & 0,0000 & 0,0000 & 0,0000 \\
$\Gamma_{0,3}\left(\xi_{2}\right)$ & $\psi_{9}$ & & $-0,0002$ & $-0,0002$ & $-0,0004$ \\
$\Gamma_{4,0}\left(\xi_{1}\right)$ & $\psi_{10}$ & & & 0,0089 & 0,0093 \\
$\Gamma_{3,1}\left(\xi_{1}, \xi_{2}\right)$ & $\psi_{11}$ & & & 0,0053 & 0,0055 \\
$\Gamma_{2,2}\left(\xi_{1}, \xi_{2}\right)$ & $\psi_{12}$ & & & 0,0000 & 0,0000 \\
$\Gamma_{1,3}\left(\xi_{1}, \xi_{2}\right)$ & $\psi_{13}$ & & & 0,0000 & 0,0000 \\
$\Gamma_{0,4}\left(\xi_{2}\right)$ & $\psi_{14}$ & & & 0,0000 & 0,0000 \\
$\Gamma_{5,0}\left(\xi_{1}\right)$ & $\psi_{15}$ & & & & $-0,0009$ \\
$\Gamma_{4,1}\left(\xi_{1}, \xi_{2}\right)$ & $\psi_{16}$ & & & & $-0,0006$ \\
$\Gamma_{3,2}\left(\xi_{1}, \xi_{2}\right)$ & $\psi_{17}$ & & & & 0,0000 \\
$\Gamma_{2,3}\left(\xi_{1}, \xi_{2}\right)$ & $\psi_{18}$ & & & & 0,0000 \\
$\Gamma_{1,4}\left(\xi_{1}, \xi_{2}\right)$ & $\psi_{19}$ & & & & 0,0000 \\
$\Gamma_{0,5}\left(\xi_{2}\right)$ & $\psi_{20}$ & & & & \\
\hline & & & & & \\
\hline
\end{tabular}

values are correctly evaluated for any order of the PCE. A PCE of order 4 is recommended and it will be used for all subsequent computations.

\section{Comparison of Failure Probability between Metamodel and Original Model}

Fig. 3(a) shows the PDF of the critical collapse pressure expressed by the nondimensional parameter $\sigma_{c} / \gamma D$ ) as provided by the analytical model and by the PCEs of orders 2, 3, 4, and 5. The failure probability corresponding to different values of the applied pressure $\sigma_{t} / \gamma D$ is then obtained from the simple formula $p_{f}=1-\mathrm{CDF}$, where CDF is the cumulative density function obtained by the integration of the PDF. The failure probability is shown in Fig. 3(b) for the practical high values of the tunnel pressure, and a log scale is used to emphasize the maximal difference between the results of the different PCEs and the solution given by the analytical model at the distribution tail. The difference between the failure probabilities provided by PCEs of orders 4 and 5 is very small (i.e., less than $4 \%$ for a pressure $\sigma_{t} / \gamma D$ of 0.4 ), and the corresponding increase in the computation time is close to $50 \%$, according to the number of calls of the model. Thus, order 4 will be used in all subsequent computations. The original analytical model requires approximately $28 \mathrm{~h}$ with a Core2 Quad CPU $2.40 \mathrm{GHz}$ PC for completing the whole sampling process. This time
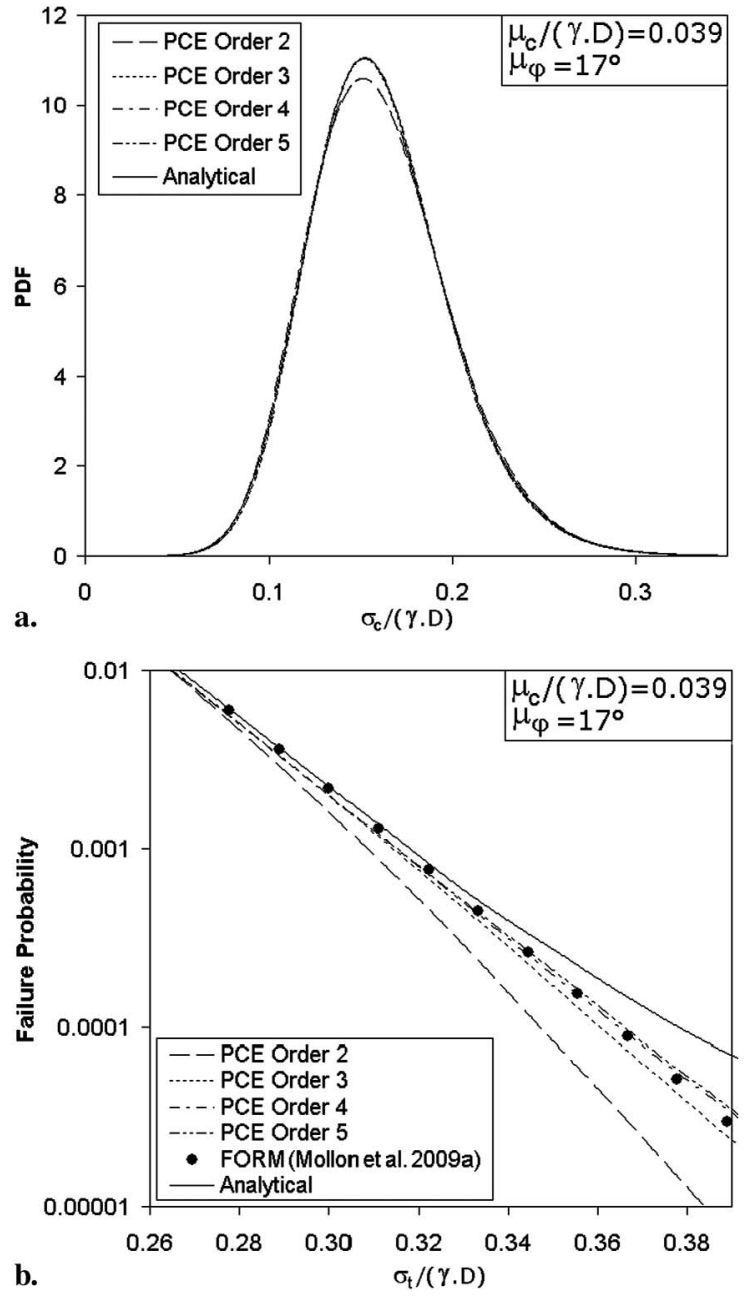

Fig. 3. Influence of the PCE order on the probabilistic results from CSRSM and comparison with the analytical results: (a) PDF of the critical collapse pressure; (b) failure probability for high values of the critical collapse pressure (distribution tail)

is significant compared to the time required for using PCE (i.e., a few seconds) because each computation of the critical pressure by the original model requires a numerical optimization that takes few seconds. This clearly shows the interest of the metamodel. The failure probabilities obtained by Mollon et al. (2009a) by using FORM with the same deterministic model are also shown in Fig. 3(b). The results obtained by CSRSM with a fourth order PCE are close to the ones obtained by FORM. This may be explained by the linearity of the limit state surface in the neighborhood of the design points.

Table 4 presents a comparison of the failure probabilities obtained by the present fourth order PCE and the ones obtained

Table 3. Statistical Moments and Sobol Indexes by PCE for Several Orders

\begin{tabular}{lcccccccc}
\hline & \multicolumn{3}{c}{ Statistical moments } & & \multicolumn{2}{c}{ Sobol indexes } \\
\cline { 2 - 3 } Order & Mean & Variance & Skewness & Kurtosis & & Friction angle $\left(\xi_{1}\right)$ & Cohesion $\left(\xi_{2}\right)$ & Friction angle and cohesion $\left(\xi_{1}, \xi_{2}\right)$ \\
\hline 2 & 28.92 & 47.90 & 0.496 & 0.409 & & 0.538 & 0.457 & 0.005 \\
3 & 28.93 & 46.12 & 0.555 & 0.703 & & 0.530 & 0.464 & 0.006 \\
4 & 28.92 & 46.00 & 0.552 & 0.744 & & 0.530 & 0.465 & 0.005 \\
5 & 28.92 & 46.02 & 0.552 & 0.749 & & 0.530 & 0.465 & 0.005 \\
\hline
\end{tabular}


Table 4. Comparison of the Failure Probabilities Obtained for PCE from an Analytical Model and for RSM from a Numerical Model

\begin{tabular}{lcc}
\hline$\sigma_{t}(\mathrm{kPa})$ & $\begin{array}{c}P_{f} \text { for PCE order } 4 \text { from an } \\
\text { analytical model }\end{array}$ & $\begin{array}{c}P_{f} \text { for RSM from a } \\
\text { numerical model }\end{array}$ \\
\hline 50 & $5.52 \times 10^{-3}$ & $3.47 \times 10^{-2}$ \\
60 & $4.24 \times 10^{-4}$ & $3.11 \times 10^{-3}$ \\
70 & $3.49 \times 10^{-5}$ & $2.31 \times 10^{-4}$ \\
\hline
\end{tabular}

by Mollon et al. (2009b) by using a response surface methodology (RSM) derived from a finite-difference numerical model. Significant differences appear between the results obtained by RSM and those obtained by the approach presented in this paper, although the trends are correct. The difference among the deterministic values of $\sigma_{c}$ given by both models for the mean values of the parameters (i.e., $\varphi=17^{\circ}$ and $c=7 \mathrm{kPa}$ ) is not significant. The analytical model provides $\sigma_{c}=28.3 \mathrm{kPa}$ and the numerical model provides $\sigma_{c}=34.5 \mathrm{kPa}$, which leads to a relative difference of $18 \%$. The values of the failure probabilities should be used with care because they are very dependent on the mechanical model error.

\section{Comparison of Response Surface between Metamodel and Original Model}

Fig. 4 presents the response surfaces provided by the analytical model, and by its PCEs for the orders 2, 3, 4, and 5 for the mean
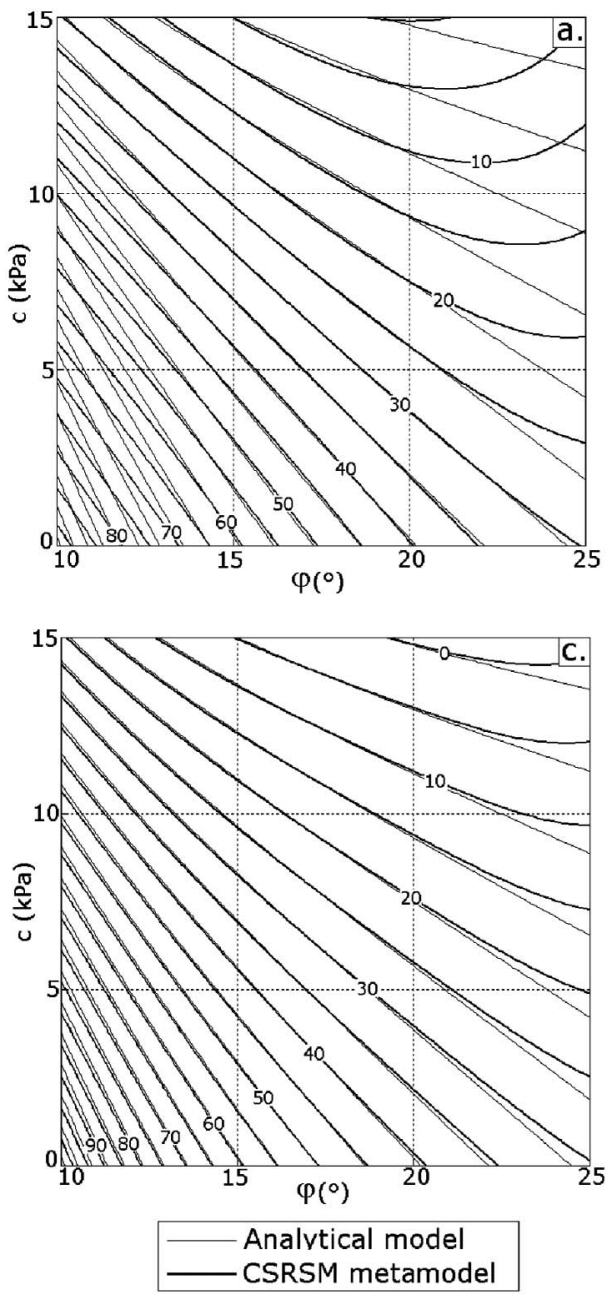

values of the soil shear strength parameters. These response surfaces are represented by lines of equal value of the critical collapse pressure $\sigma_{c}$ in the physical $(\varphi, c)$ space. The response surfaces given by PCEs of orders 2 and 3 seem to be quite different from the analytical one, except in the central zone (i.e., in the zone where the collocation points of these PCE are located). The orders 4 and 5 are much better, especially in the remote zones of the physical space (i.e., corresponding to very high or low values of $c$ and/or $\varphi$ ). This is because the area covered by the collocation points of these orders is more extended. This fact explains why orders 4 and 5 provide a better approximation of the analytical model at the distribution tails.

\section{Sensitivity Analysis}

This section focuses on a sensitivity analysis showing the effect of the probabilistic characteristics of the two random variables (i.e., COV, correlation, type of probability distribution) on both (1) the distribution of the critical collapse pressure; and (2) the failure probabilities corresponding to different practical values of the tunnel applied pressure (i.e., at the distribution tail). The reference case extensively studied in the previous section is also considered. This study will be performed with the help of CSRSM by using a fourth order PCE as a metamodel. A Monte Carlo-simulation with
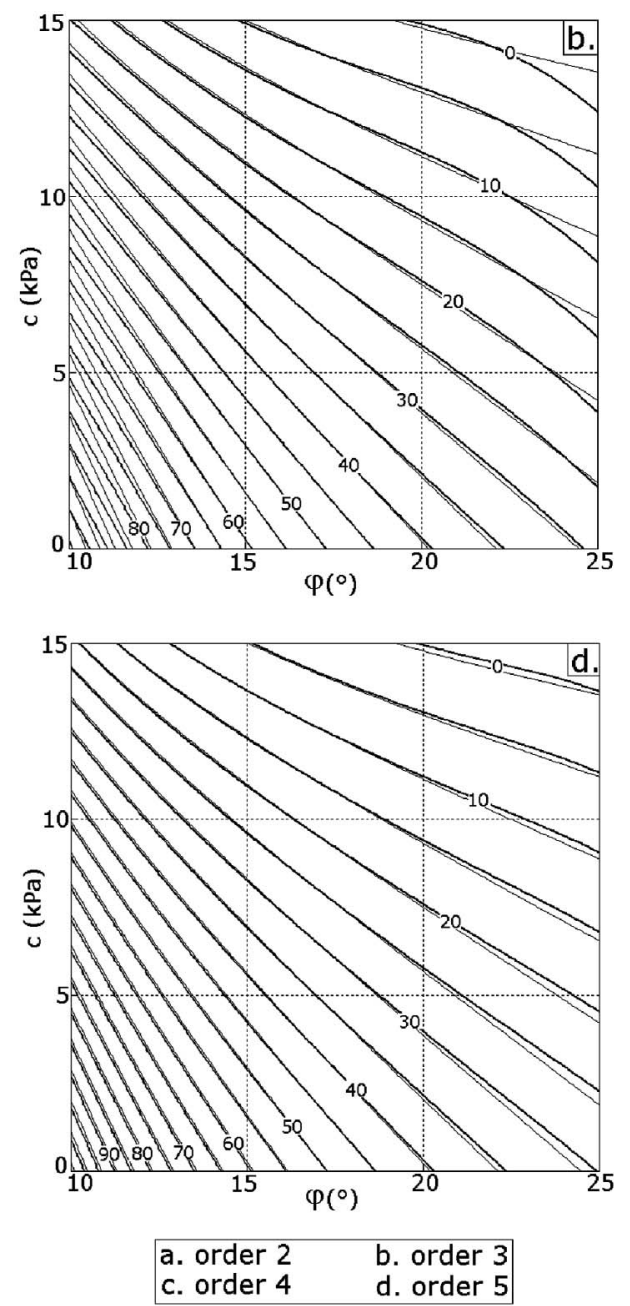

Fig. 4. Response surfaces of the critical collapse pressure (lines of equal value of $\sigma_{c}$ in $\mathrm{kPa}$ ) in the $(\varphi, c)$ plane as given by the analytical mechanism and the PCE for several orders 

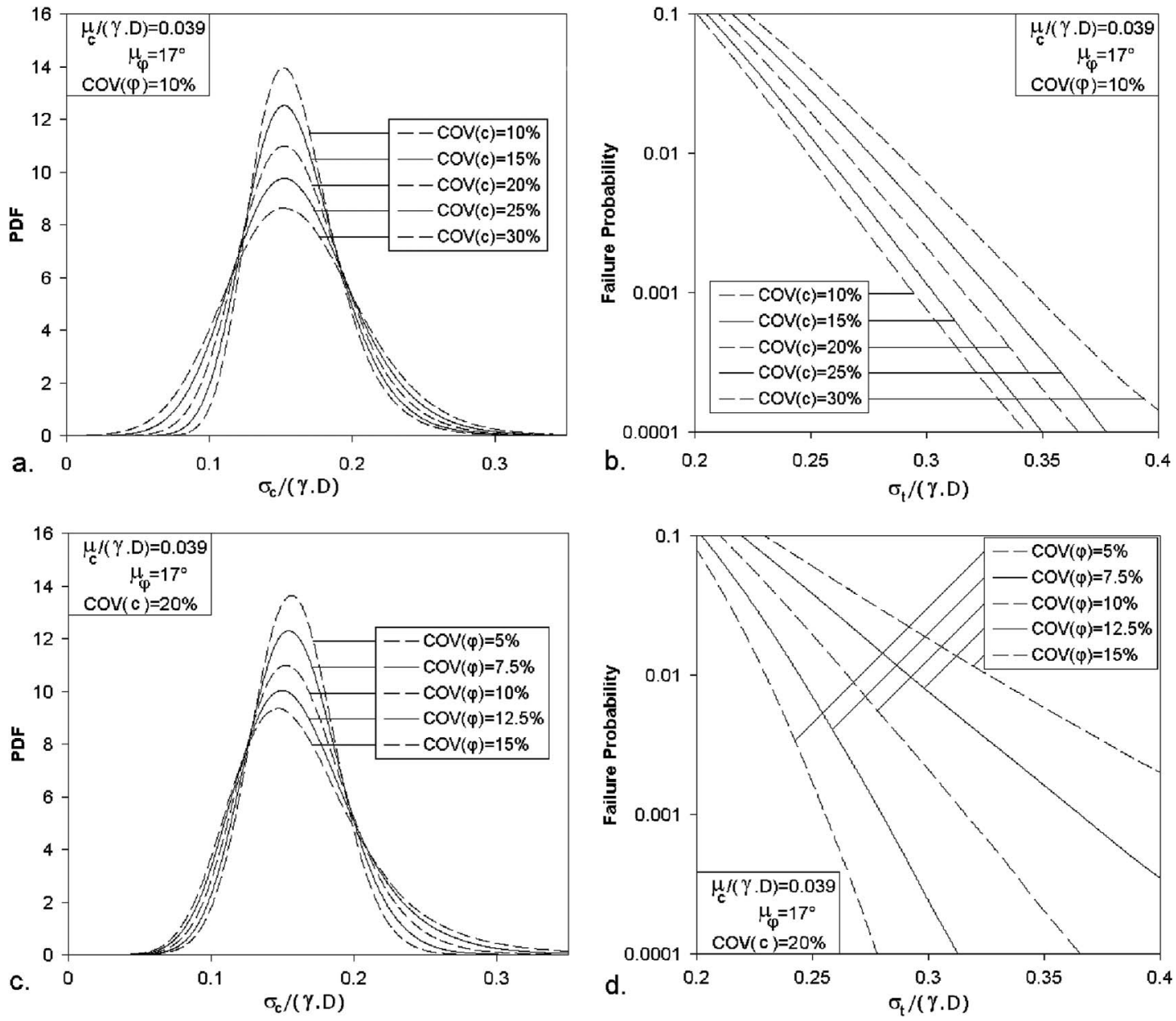

Fig. 5. Influence of the coefficients of variation of the random variables on the PDF of the critical collapse pressure: (a) influence of $\operatorname{COV}(\varphi)$; (b) influence of $\operatorname{COV}(c)$
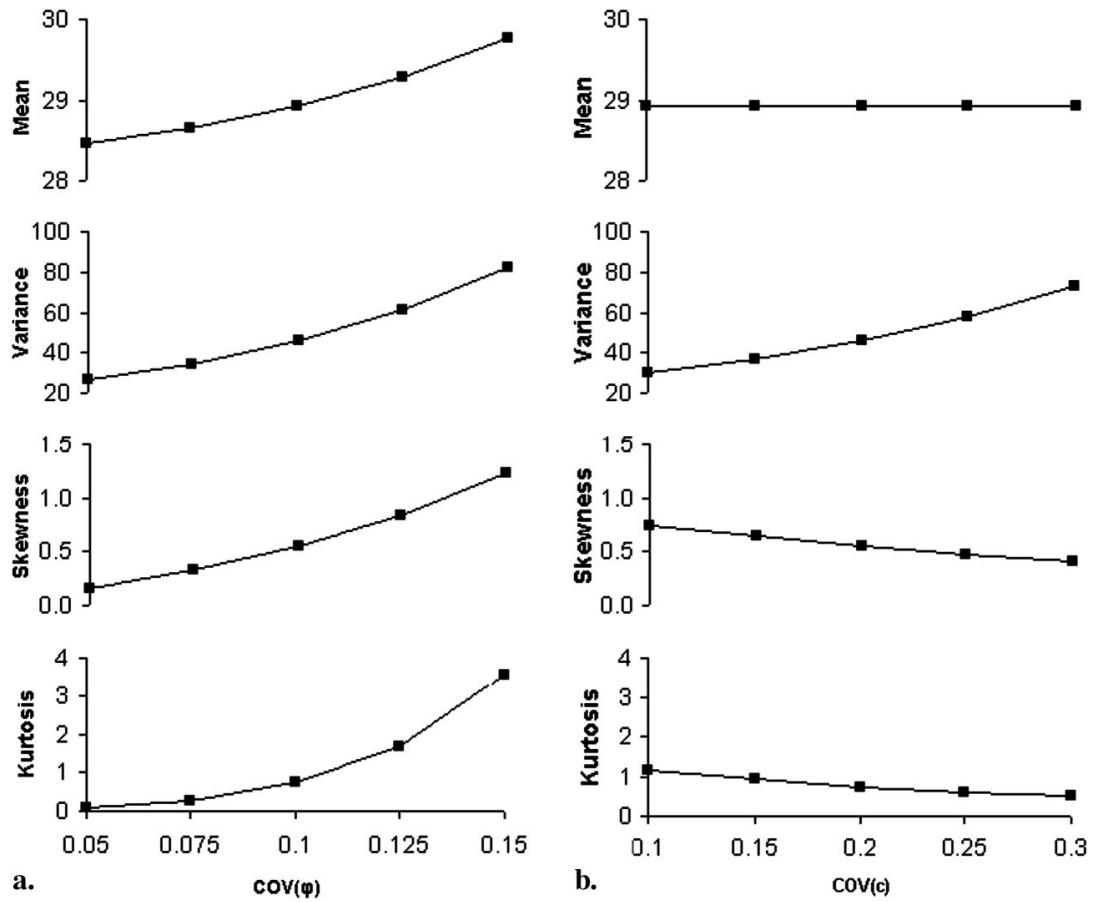

Fig. 6. Influence of the coefficients of variation of $\varphi$ and $c$ on the first four statistical moments of the critical collapse pressure: (a) influence of $\operatorname{COV}(\varphi) ;(b)$ Influence of $\operatorname{COV}(c)$ 
$1,000,000$ samples was used in the subsequent sections for the probabilistic analysis.

\section{Effect of Coefficients of Variation of Random Variables}

The influence of the coefficients of variation of $\varphi$ and $c$ was studied in Fig. 5. In Figs. 5(a) and 5(c), an increase in the COV of one of the random variables leads to a more widespread PDF curve, whereas a small dispersion of a random variable increases the peak of the PDF. A change in $\operatorname{COV}(\varphi)$ modifies the value of the collapse pressure corresponding to the maximal value of the PDF (i.e., the mode), which is not true for $\operatorname{COV}(c)$. Figs. 5(b) and 5(d) show the effect of the coefficients of variation of the two variables on the probabilities of failure for high values of the parameter $\sigma_{t} /(\gamma . D)$. It appears that a given variation (percent increase with respect to the reference value) in $\operatorname{COV}(\varphi)$ has a much larger effect on the failure probability than a similar variation in $\operatorname{COV}(c)$.

By using PCE coefficients, the values of the first four statistical moments of the response were computed analytically by using Eqs. (8)-(11). The effect of $\operatorname{COV}(\varphi)$ and $\operatorname{COV}(c)$ on the mean, variance, skewness, and kurtosis of the distribution of the critical collapse pressure is shown in Fig. 6. Fig. 6 shows a very similar influence of the two COVs on the mean and variance of the critical collapse pressure: almost no effect exists on the mean, particularly for $\operatorname{COV}(c)$; however, the variance of the response increases with the increase of COV. This is consistent with the observation of the PDFs shown in Fig. 5. Although the variance of the response increases with the $\mathrm{COV}$ of each of the random variables, this variance is less sensitive to $\operatorname{COV}(c)$ than to $\operatorname{COV}(\varphi)$. For the practical case in which $\operatorname{COV}(\varphi)=10 \%$ and $\operatorname{COV}(c)=20 \%$, the two random variables seem to have an equal weight on the dispersion of the response because of the large variability of $c$ compared to that of $\varphi$. Fig. 6 also show the effect of $\operatorname{COV}(\varphi)$ and $\operatorname{COV}(c)$ on the skewness and kurtosis of the response. The coefficient of variation of the friction angle appears to have a strong effect on these two statistical moments. For a small value of $\operatorname{COV}(\varphi)$, the skewness and kurtosis of the response are almost null, which indicates that the probability distribution of the response is not far from a Gaussian one. These moments however, take high values when $\operatorname{COV}(\varphi)$ increases, which indicates that the shape of the probability distribution of the output changes (i.e., the point of maximum density of probability; the mode moves to smaller values). On the contrary, $\operatorname{COV}(c)$ only has a small effect on the skewness and kurtosis of the probability distribution of the output, which indicates that the shape of this distribution will not change a lot when increasing $\operatorname{COV}(c)$, except for its variance, of course. These remarks are consistent with the observation of the PDFs provided in Fig. 5, in which the shape of the distribution changes with the increase of $\operatorname{COV}(\varphi)$ and remains quite the same with the increase of $\operatorname{COV}(c)$.

Fig. 7 presents the effect of $\operatorname{COV}(\varphi)$ and $\operatorname{COV}(c)$ on the Sobol indexes computed analytically from the PCE coefficients by using Eq. (13). Because the sum of all the Sobol indexes is always equal to 1 , a convenient method was adopted to represent them on a single graph: the upper part in light gray represents the Sobol index of $c$ [called SU $(c)$ ], the lower part in dark gray represents the Sobol index of $\varphi$ [called $\operatorname{SU}(\varphi)$ ], and the middle part in white represents the Sobol index of the group of variables $(c, \varphi)$ [called $\mathrm{SU}(c, \varphi)$ ]. The latter is almost equal to zero and can be neglected in this study. Therefore, $\mathrm{SU}(\varphi)+\mathrm{SU}(c) \approx 1$. For the practical values of COVs [i.e., $\operatorname{COV}(\varphi)=10 \%$ and $\operatorname{COV}(c)=20 \%$ ], the Sobol indexes of the two variables (Fig. 7) are not far from 50\%. This indicates that the two random variables seem to have an equal weight on the response. This result conforms with that obtained from Fig. 6. Fig. 7 show that the increase of the COV of one of the variables
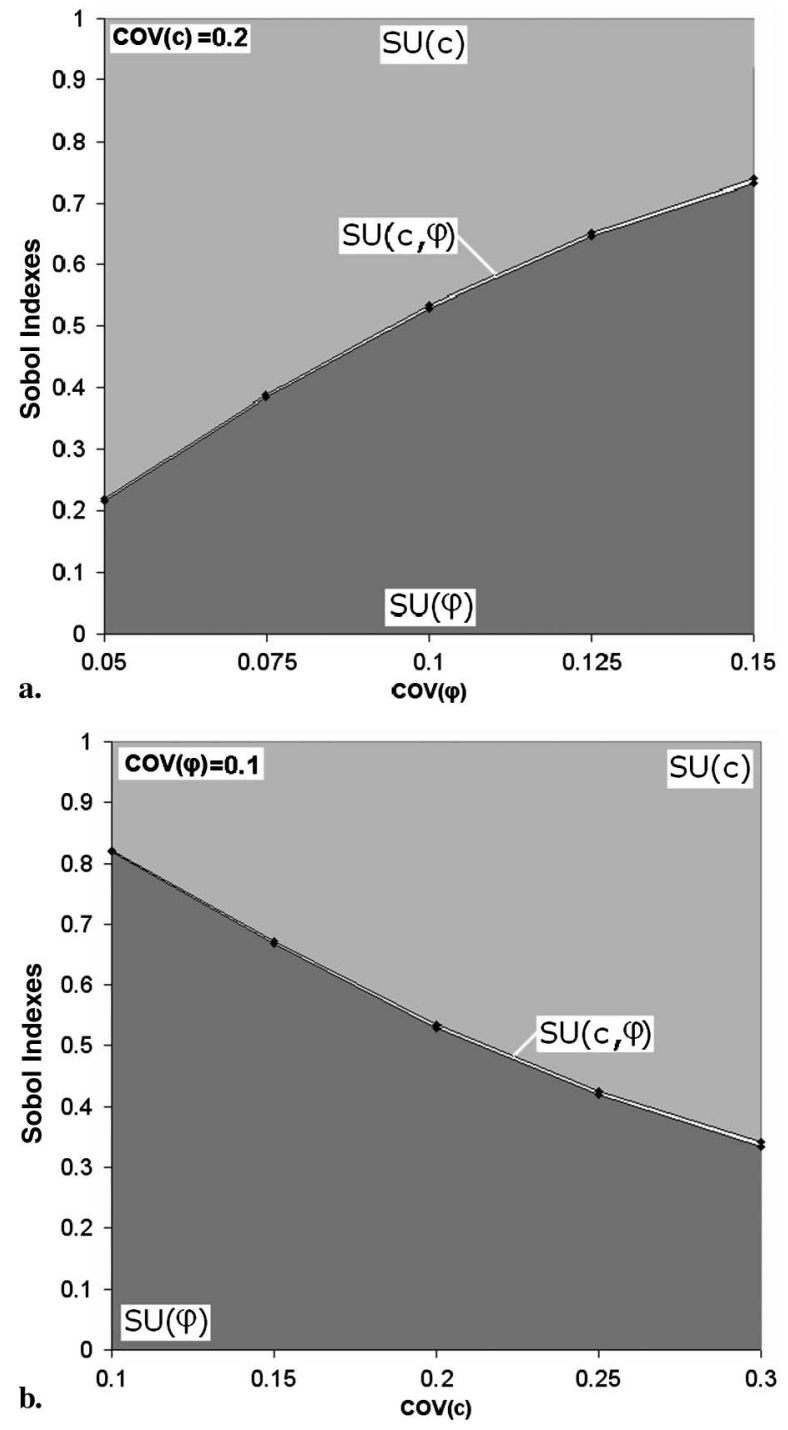

Fig. 7. Influence of the coefficients of variation of $\varphi$ and $c$ on the Sobol indexes: (a) influence of $\operatorname{COV}(\varphi)$; (b) influence of $\operatorname{COV}(c)$

logically induces an increase in the Sobol index of this variable and subsequently, its weight in the variability of the response. For example, in the case of a high COV of the friction angle (i.e., $\operatorname{COV}(\varphi)=15 \%$ ), its Sobol index reaches $70 \%$ and the one of $c$ decreases to $30 \%$.

\section{Effect of Nonnormality of Random Variables}

To account for the nonnormal distributions of random variables, the cohesion and the angle of internal friction are assumed to follow a lognormal and a beta distribution, respectively. The parameters of these probability distributions are determined from the means and standard deviations of $c$ and $\varphi$, respectively. Two sets of the coefficients of variation are studied in this paper. The standard COVs correspond to the reference case [i.e., $\operatorname{COV}(\varphi)=10 \%$ and $\operatorname{COV}(c)=20 \%$ ], and the high COVs correspond to these values increased by $50 \%$ [i.e., $\operatorname{COV}(\varphi)=15 \%$ and $\operatorname{COV}(c)=30 \%$ ]. Fig. 8(a) compares the PDFs corresponding to normal and nonnormal variables for the two sets of the coefficients of variation, and Fig. 8(b) compares the corresponding failure probabilities for high values of $\sigma_{t} /(\gamma . D)$; that is, at the distribution tail. For the two sets of COVs, nonnormality of the probability distributions of the input variables seems to have a small effect on the general shape of 

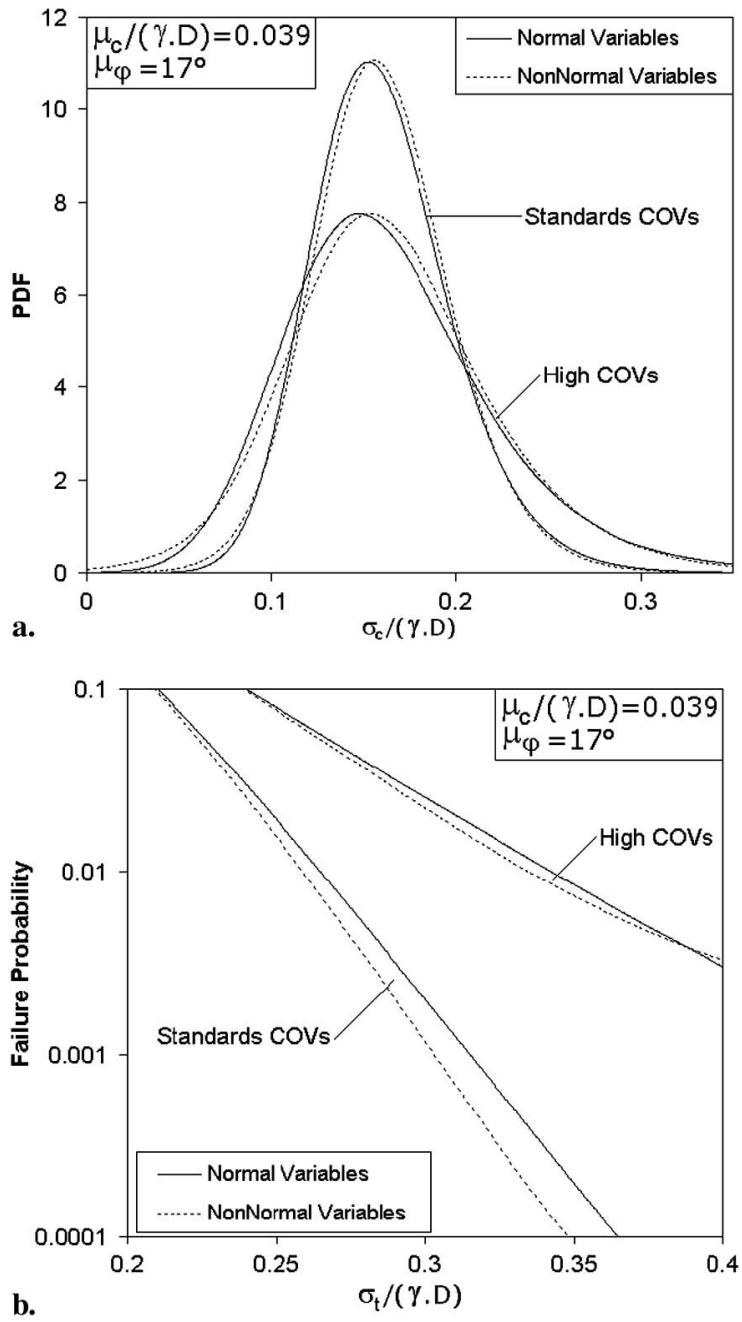

Fig. 8. Influence of the nonnormality of $\varphi$ and $c$ on the PDF of the critical collapse pressure for two different sets of COVs

the distribution of the response and on the failure probabilities at the distribution tail.

\section{Effect of Correlation among Random Variables}

It is generally believed that a negative correlation exists between the friction angle and the cohesion (Cherubini 2000). However, further experimental tests are needed to confirm this statement. The influence of the correlation coefficient in the range $-0.6 \leq \rho_{c \varphi} \leq$ 0.6 was studied in this paper. Fig. 9(a) shows the PDFs of the critical collapse pressure for several values of $\rho_{c \varphi}$. It appears that the correlation has a strong influence on the dispersion of the response. A negative correlation leads to an accentuated peak of the PDF; a positive correlation leads to a widespread distribution. On the other hand, Fig. 9(b) shows the probabilities of failure at the distribution tail for different values of the correlation coefficient. A positive correlation between $c$ and $\varphi$ increases the failure probability. Fig. 10 presents the first four statistical moments of the response against $\rho_{c \varphi}$ as computed analytically from the fourth-order PCE coefficients. The coefficient of correlation has a small effect on the mean value of the response, but it has a significant effect on its variability. The variance of the critical collapse pressure is, for example, four times larger for $\rho_{c \varphi}=0.6$ than that of $\rho_{c \varphi}=-0.6$. On the other hand, both the skewness and the kurtosis of the response slightly increase with the correlation of the input variables.
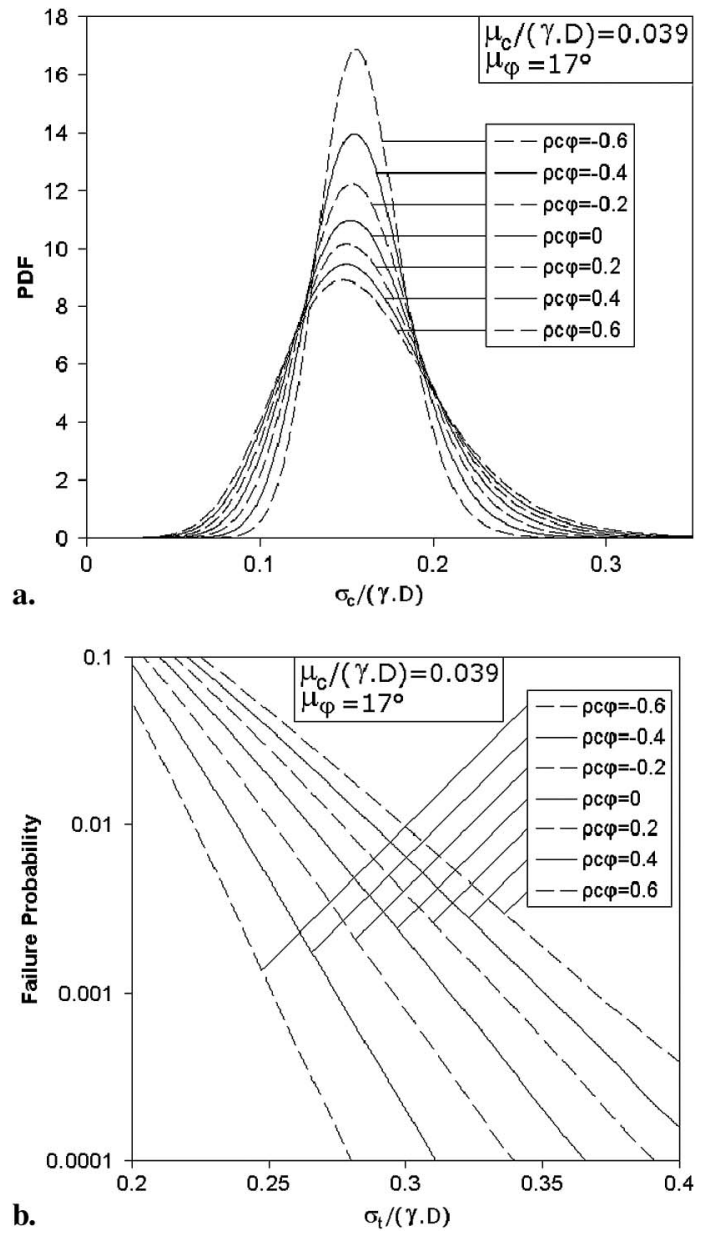

Fig. 9. Influence of the correlation coefficient between $\varphi$ and $c$ on the PDF of the critical collapse pressure
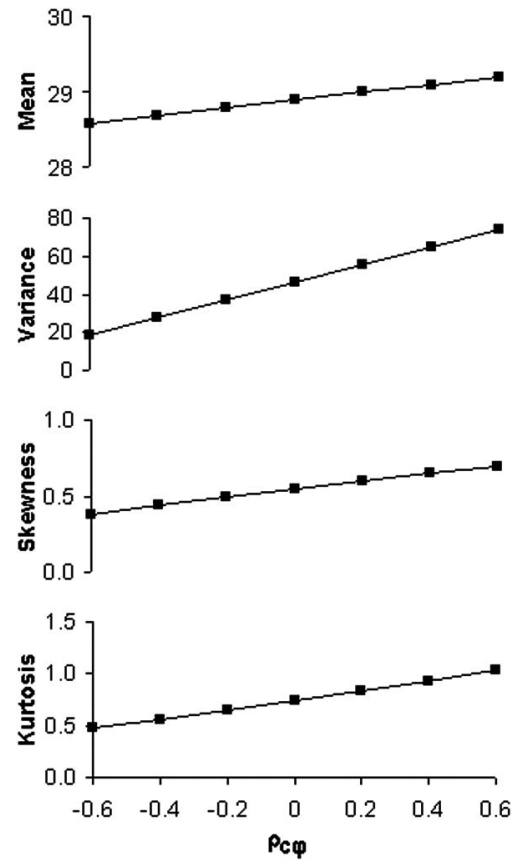

Fig. 10. Influence of the correlation coefficient between $\varphi$ and $c$ on the first four statistical moments of the critical collapse pressure 


\section{Conclusion}

The CSRSM was applied in this paper to resolve a probabilistic analysis of the face stability of a circular tunnel driven by a pressurized shield. The uncertain parameters considered in the analysis were the soil shear strength properties; namely, the friction angle $\varphi$ and the cohesion $c$. An analytical kinematical limit analysis model was used as a deterministic model. For a given set of the probabilistic parameters of the input variables, CSRSM consisted of replacing the deterministic model by an analytical metamodel, defined by a polynomial chaos expansion (PCE). The unknown coefficients of this PCE were obtained by regression after the computation of the response of the model at a given number of collocation points. It was shown that

- A fourth-order PCE is sufficient for the CSRSM metamodel to simulate the system response with an acceptable accuracy;

- The coefficients of variation of $c$ and $\varphi$ have a great effect on the distribution of the critical collapse pressure. An increase in the $\mathrm{COV}$ of one of the random variables leads to a more widespread PDF, whereas a small dispersion of a random variable increases the peak of this PDF. The COV of $\varphi$ has a much greater effect on the failure probabilities than the one of $c$;

- For the practical case in which $\operatorname{COV}(\varphi)=10 \%$ and $\operatorname{COV}(c)=$ $20 \%$, both $\varphi$ and $c$ have a similar probabilistic effect on the variability of the critical collapse pressure, although the variance of the response is less sensitive to a variation in the cohesion. This observation was explained by the high variability of $c$ compared to that of $\varphi$;

- The assumption of nonnormal distributions for the random variables, which is necessary to more accurately simulate these variables, does not have a great effect on the distribution of the critical collapse pressure and on the failure probability;

- The correlation between $c$ and $\varphi$ has a great effect on the variability of the response. A negative correlation strongly reduces the probability of failure of the tunnel face, whereas a positive correlation is conservative and could lead to noneconomical designs. Therefore, the characterization of a possible correlation between $c$ and $\varphi$ should be carefully investigated; and

- Although the deterministic model used in this study is not perfectly accurate, its use in the framework of CSRSM provides a satisfactory estimate of the probabilities of failure in terms of the trends, for a very small time cost. However, a precise determination of the failure probabilities cannot be achieved without the use of a very accurate deterministic model, such as a more complex analytical model or a numerical model.

\section{Appendix I. Multivariate Hermite Polynomials}

The univariate Hermite polynomials are defined by the following recurrence formula:

$$
\mathrm{He}_{0}(\xi)=1 ; \quad \mathrm{He}_{n+1}(\xi)=\xi \cdot \mathrm{He}_{n}(\xi)-n \cdot \mathrm{He}_{n-1}(\xi)
$$

Thus, the first univariate Hermite polynomials are

$$
\operatorname{He}_{1}(\xi)=\xi
$$

$$
\begin{gathered}
\mathrm{He}_{2}(\xi)=\xi^{2}-1 \\
\mathrm{He}_{3}(\xi)=\xi^{3}-3 \xi \\
\mathrm{He}_{4}(\xi)=\xi^{4}-6 \xi^{2}+3 \\
\operatorname{He}_{5}(\xi)=\xi^{5}-10 \xi^{3}+15 \xi
\end{gathered}
$$

A multivariate Hermite polynomial is defined as the product of several univariate Hermite polynomials of different variables. For $n$ variables, its expression is given by

$$
\Gamma_{i 1, i 2, \ldots, i n}\left(\xi_{1}, \xi_{2}, \ldots, \xi_{n}\right)=\operatorname{He}_{i 1}\left(\xi_{1}\right) \cdot \mathrm{He}_{i 2}\left(\xi_{2}\right) \cdot \ldots \cdot \mathrm{He}_{i n}\left(\xi_{n}\right)
$$

In this paper, only two variables were used, and the expression of the bivariate Hermite polynomials used in Table 1 is

$$
\Gamma_{i, j}\left(\xi_{1}, \xi_{2}\right)=\operatorname{He}_{i}\left(\xi_{1}\right) \cdot \operatorname{He}_{j}\left(\xi_{2}\right)
$$

For a simple use in mathematical formulas, the multivariate Hermite polynomials are often renamed and sorted by using only one numerical index, for example

$$
\Gamma_{i, j}\left(\xi_{1}, \xi_{2}\right)=\psi_{k}
$$

The $p$ polynomials $\psi_{k}$ of order $(i+j) \leq n$ form the basis of the bivariate PCE of order $n$. An example of the numbering of these polynomials is given in Table 2 .

\section{Appendix II. Computation of the Expectation of Products of Bivariate Hermite Polynomials}

An analysis of bivariate Hermite polynomials is included in this appendix. A complete development of this computation for Hermite polynomials of more than two variables can be found in Sudret et al. (2006). Each polynomial $\psi_{i}$ of the basis of the PCE of two variables $\xi_{1}$ and $\xi_{2}$ can be entirely defined by two indexes $i_{1}$ and $i_{2}$ (see Table 2) such that

$$
\psi_{i}=\Gamma_{i 1, i 2}\left(\xi_{1}, \xi_{2}\right)=\operatorname{He}_{i 1}\left(\xi_{1}\right) \cdot \operatorname{He}_{i 2}\left(\xi_{2}\right)
$$

With this notation, it can be proved that

$$
\mathrm{E}\left(\psi_{i}^{2}\right)=i_{1} ! i_{2} !
$$

$$
\begin{gathered}
\mathrm{E}\left(\psi_{i} \cdot \psi_{j} \cdot \psi_{k}\right)=\mathrm{D}_{i 1, j 1, k 1} \cdot \mathrm{D}_{i 2 ; j 2, k 2} \\
\mathrm{E}\left(\psi_{i} \cdot \psi_{j} \cdot \psi_{k} \cdot \psi_{l}\right)=\mathrm{D}_{i 1, j 1, k 1, l 1} \cdot \mathrm{D}_{i 2 ; j 2, k 2, l 2}
\end{gathered}
$$

In these expressions, the $\mathrm{D}$ terms are obtained by

$$
\mathrm{C}_{i, j, k}= \begin{cases}(i ! j !) /\{[(i+j-k) / 2] ![(j+k-i) / 2] ![(k+i-j) / 2] !\} & \text { if }\left\{\begin{array}{l}
(i+j+k) \text { even } \\
k \epsilon[|i-j|, i+j]
\end{array}\right. \\
0 & \text { otherwise }\end{cases}
$$




$$
\begin{gathered}
\mathrm{D}_{i, j, k}=\mathrm{C}_{i, j, k} \cdot k ! \\
\mathrm{D}_{i, j, k, l}=\sum_{q \geq 0} \mathrm{D}_{i, j, q} \cdot \mathrm{C}_{k, l, q}
\end{gathered}
$$

\section{References}

Anagnostou, G., and Kovari, K. (1996). "Face stability conditions with earth-pressure-balanced shields." Tunnelling Underground Space Technol., 2(11), 165-173.

Augarde, C. E., Lyamin, A. V., and Sloan, S. W. (2003). "Stability of an undrained plane strain heading revisited." Comput. Geotech., 30(5), 419-430.

Berveiller, M., Sudret, B., and Lemaire, M. (2006). "Stochastic finite elements: A non intrusive approach by regression." Revue Européenne de Mécanique Numérique, 1-2-3(15), 81-92.

Broms, B. B., and Bennermark, H. (1967). "Stability of clay at vertical openings." J. Soil Mech. and Found. Div., 193(SM1), 71-94.

Chambon, P., and Corté, J. F. (1994). "Shallow tunnels in cohesionless soil: Stability of tunnel face." J. Geotech. Eng., 120(7), 1148-1165.

Chen, W. F., (1975). Limit analysis and soil plasticity, Elsevier Scientific, London, 637.

Chen, W. F., and Liu, X. L. (1990). Limit analysis in soil mechanics, Elsevier Science, Amsterdam, The Netherlands.

Cherubini, C. (2000). "Reliability evaluation of shallow foundation bearing capacity on C', $\varphi^{\prime}$ soils." Can. Geotech. J., 37(1), 264-269.

Davis, E. H., Gunn, M. J., Mair, R. J., and Seneviratne, H. N. (1980). “The stability of shallow tunnels and underground openings in cohesive material." Géotechnique, 30(4), 397-416.

Eisenstein, A. R., and Ezzeldine, O. (1994). "The role of face pressure for shields with positive ground control." Tunneling and ground conditions, Balkema, Rotterdam, The Netherlands, 557-571.

Ellstein, A. R. (1986). "Heading failure of lined tunnels in soft soils." Tunnels Tunnelling, 18, 51-54.

Huang, S. P., Liang, B., and Phoon, K. K. (2009). "Geotechnical probabilistic analysis by collocation-based stochastic response surface method: An Excel add-in implementation." Georisk, 3(2), 75-86.
Isukapalli, S. S. (1999). "An uncertainty analysis of transport-transformation models." Ph.D. thesis, The State Univ. of New Jersey, New Brunswick, NJ.

Isukapalli, S. S., Roy, A., and Georgopoulos, P. G. (1998). "Stochastic response surface methods (SRSMs) for uncertainty propagation: Application to environmental and biological systems." Risk Anal., 18(3), 351-363.

Klar, A., Osman, A. S., and Bolton, M. (2007). "2D and 3D upper bound solutions for tunnel excavation using 'elastic' flow fields." Int. J. Numer. Anal. Meth. Geomech., 31(12), 1367-1374.

Leca, E., and Dormieux, L. (1990). "Upper and lower bound solutions for the face stability of shallow circular tunnels in frictional material." Géotechnique, 40(4), 581-606.

Mollon, G., Dias, D., and Soubra, A.-H. (2009a). "Probabilistic analysis and design of circular tunnels against face stability." Int. J. Geomech., 9(6), 237-249.

Mollon, G., Dias, D., and Soubra, A.-H. (2009b). "Probabilistic analysis of circular tunnels in homogeneous soils using response surface methodology." J. Geotech. Geoenviron. Eng., 135(9), 1314-1325.

Mollon, G., Dias, D., and Soubra, A.-H. (2010). "Face stability analysis of circular tunnels driven by a pressurized shield." J. Geotech. Geoenviron. Eng., 1(136), 215-229.

Phoon, K. K., and Huang, S. P. (2007). "Geotechnical probabilistic analysis using collocation-based stochastic response surface method." Applications of statistics and probability in civil engineering, J. Kanda, T. Takada, and H. Furada, eds., Taylor and Francis, London.

Sudret, B. (2008). "Global sensitivity analysis using polynomial chaos expansion.” Reliab. Eng. Syst. Saf., 93(7), 964-979.

Sudret, B., Berveiller, M., and Lemaire, M. (2006). "A stochastic finite element procedure for moment and reliability analysis." Eur. J. Comput. Mech., 7-8(15), 825-866.

Youssef Abdel Massih, D. S., and Soubra, A.-H. (2008). "Reliability-based analysis of strip footings using response surface methodology." Int. J. Geomech., 8(2), 134-143.

Youssef Abdel Massih, D. S., Soubra, A.-H., and Low, B. K. (2008). "Reliability-based analysis and design of strip footings against bearing capacity failure." J. Geotech. Geoenviron. Eng., 134(7), 917-928. 